\title{
ULTRAFAST ELECTRON EJECTION AND TRAPPING IN SEMICONDUCTOR COLLOIDS AFTER MULTIPLE PHOTON ABSORPTION
}

\author{
M. KASCHKE ', N.P. ERNSTING \\ Abteilung Laserphysik, Max-Planck-Institut für Biophysikalische Chemie, \\ Postfach 2481, D-3400 Göttingen, Federal Republic of Germany \\ U. MÜLLER and H. WELLER \\ Bereich Strahlenchemie, Hahn-Meitner-Institul Berlin, D-1000 Berlin 39, Germany
}

Received 5 February 1990

\begin{abstract}
Quantum-size semiconductor microcrystallites of ZnCdS and CdS in aqueous and isopropanol solution were optically excited using intense sub-picosecond UV laser pump pulses. Broad-band optical probing monitored the yield of electron emission and the build-up of primary photochemical products. With typical pump fluences of femtosecond light pulses multiple photons are absorbed by one quantum-dot leading to a quantum yield of up to 0.3 of photogenerated electrons. The yield curves, as a function of pump fluence, are described by a simple kinetic model which involves the trapping-rate constant and a maximum number of electrons which can be emitted per particle. This model is shown to be consistent with thermodynamic considerations.
\end{abstract}

\section{Introduction}

The non-linear optical properties of direct-gap semiconductors have been examined intensively over the past few years, both with bulk materials [1-5] as well as microcrystals [6-17] embedded in a glass host. A relatively young class of materials consists of very small crystallites, with diameters ranging up to $100 \AA$. These "microcrystallites" are usually prepared by chemical reaction, either in a polymer film [11,18] or as a colloid from solution [19-21]. If the particle radius is less than the excitonic radius in the bulk material we speak of quantum-size microcrystallites or quantum-dots [22]. For the description of such tiny particles a molecular approach is more appropriate than a semiconductor one; however, standard pictures of semiconductor theory - such as excitons and band filling - are frequently applied. In quantum-dots the electronic wavefunctions of an electron and hole always overlap appreciably due to

1 On leave from the Zentralinstitut für Optik und Spektroskopie, Akademie der Wissenschaften der DDR, DDR-1 199 Berlin, German Democratic Republic. the confinement. In this sense, the lowest state of an electron and hole may be called an "exciton" [22].

In a previous paper [23] we have investigated the bleaching kinetics after absorption of multiple photons from an intense ultrashort light pulse at $308 \mathrm{~nm}$. It was shown that recombination of charge carriers takes place on a picosecond time scale and is fluence dependent. Further, the occurrence of rapid surface trapping of charge carriers, as well as the ultrafast ejection of electrons out of the quantum-dot in the femtosecond time-scale was established.

In this paper we examine quantum-size $\mathrm{CdS}$ as a colloid in water and in isopropanol, and compare it to colloidal $\mathrm{Zn}_{1-x} \mathrm{Cd}_{x} \mathrm{~S}$.

We report

(i) transient spectra in the range 370 to $750 \mathrm{~nm}$ for quantum-dots in water and isopropanol,

(ii) the relative yield of photoproducts absorbing in the red spectral range - mainly solvated electrons - as a function of pump fluence, solvent and particle size.

We present a simple model, which consistently explains the experimental findings for both quantumsize systems under investigation. 


\section{Experimental}

\subsection{Sample preparation}

\subsubsection{Aqueous colloids}

Aqueous stack solutions were prepared of $\mathrm{Zn}\left(\mathrm{ClO}_{4}\right)_{2}$ and of $\mathrm{Cd}\left(\mathrm{ClO}_{4}\right)_{2}$ (both 0.1 molar) and of sodium polyphosphate (Riedel-deHaën; 0.1 molar assuming a MW of 609). The synthesis was carried out in a $2 \ell$ flask sealed against air, and fitted with a septum, a pH electrode, and a glass fritte for bubbling with argon.

$\mathrm{ZnCdS}$. To $352 \mathrm{ml}$ of water in the flask were added $22.80 \mathrm{ml}$ of the $\mathrm{Zn}$ solution, $1.20 \mathrm{ml}$ of the $\mathrm{Cd}$ solution, and $24 \mathrm{ml}$ of the polyphosphate solution. The solution was degassed thoroughly for $5 \mathrm{~min}$ by bubbling with argon. Then the $\mathrm{pH}$ was adjusted to $\mathbf{1 1 . 0}$ using $1 \mathrm{~N} \mathrm{NaOH}$ solution, and argon bubbling was resumed for at least $20 \mathrm{~min}$. Finally, $42 \mathrm{ml}$ of gaseous $\mathrm{H}_{2} \mathrm{~S}$ was quickly injected into the volume above the solution, and the flask was vigorously shaken for $15 \mathrm{~s}$.

$C d S$. To $500 \mathrm{ml}$ of water in the $2 \mathrm{l}$ flask were added $4.5 \mathrm{ml}$ of the polyphosphate solution and $9 \mathrm{ml}$ of the $\mathrm{Cd}$ solution. The $\mathrm{pH}$ was adjusted to 9.7 using $\mathrm{NaOH}$ solution. $10.2 \mathrm{ml}$ of gaseous $\mathrm{H}_{2} \mathrm{~S}$ was quickly injected. After $6 \mathrm{~s}$ of reaction the flask was vigorously shaken for $3 \mathrm{~s}$. The solution was then filled into a beaker and $10 \mathrm{ml}$ of 0.2 molar $\mathrm{NaOH}$ solution was added. The beaker was heated to $90^{\circ} \mathrm{C}$ and the temperature was maintained for $3 \mathrm{~h}$. Finally, the solution was concentrated to $250 \mathrm{ml}$ in an evaporator.

\subsubsection{Alcoholic colloids}

$C d S$. A stock solution of $\mathrm{Cd}\left(\mathrm{ClO}_{4}\right)_{2}(0.1$ molar $)$ in isopropanol was prepared. To $200 \mathrm{ml}$ of isopropanol in a $250 \mathrm{ml}$ measuring flask $3.1 \mathrm{ml}$ of this stock solution were added. The flask was fitted with a septum, $8 \mathrm{ml}$ of gaseous $\mathrm{H}_{2} \mathrm{~S}$ were quickly injected into the volume above the solution, and the flask was vigorously shaken for $20 \mathrm{~s}$. The sample was then aged overnight and concentrated to $50 \mathrm{ml}$ in a rotating evaporator. The concentrated colloidal solution was stable up to $30 \mathrm{~min}$ at room temperatures, after which precipitation occurred.

\subsection{The laser system}

The pump/probe-absorption (gain) experiment used a laser system combining an excimer laser with a colliding-pulse-mode (CPM) dye laser which is described in detail in ref. [24].

\section{Results}

The absorption spectra of colloidal CdS in water and isopropanol and aqueous colloidal $\mathrm{Zn}_{0.95} \mathrm{Cd}_{0.05} \mathrm{~S}$ are showin in fig. 1. Also given in the figure is the particle diameter as a function of the wavelength of the first "excitonic" transition, according to quan-
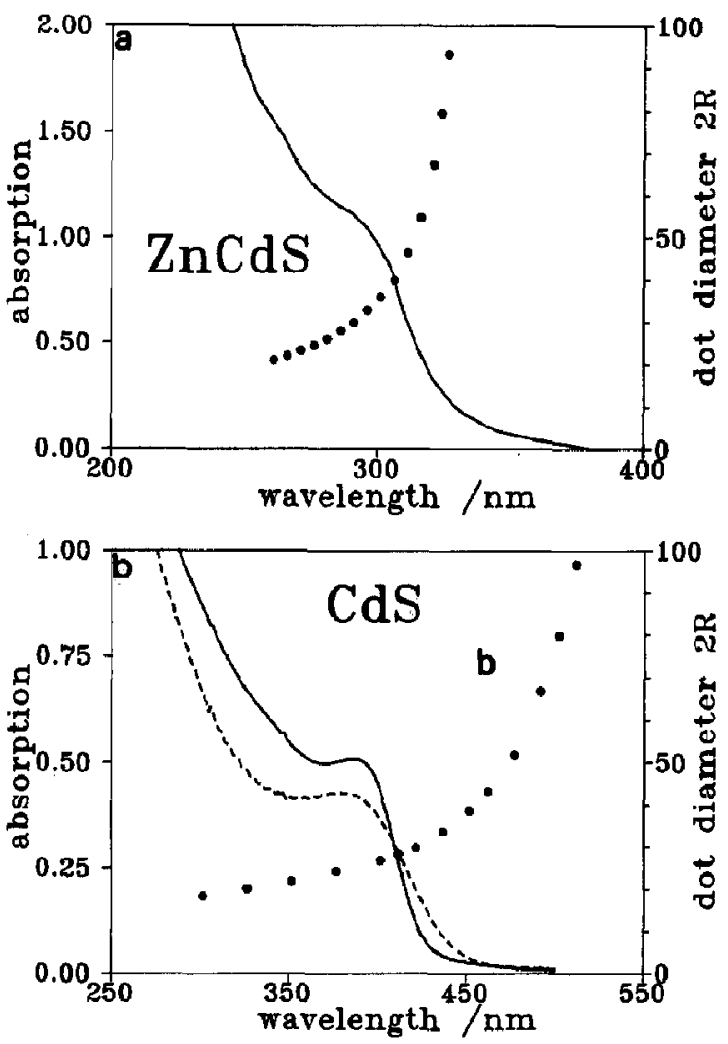

Fig. 1. (a) Solid line: absorption spectrum of aqueous colloidal $\mathrm{Zn}_{0.95} \mathrm{Cd}_{0.05} \mathrm{~S}$. Dots: particle diameter estimated from the spectral location of the "exciton". band [25]. Sample thickness for this measurement was $0.2 \mathrm{~cm}$. (b) Solid line: absorption spectrum of aqueous colloidal CdS. Dashed line: absorption spectrum of colloidal CdS in isopropanol. Dotted line: particle diameter estimated from the spectral location of the "exciton" band [25]. Sample thickness for this measurement was $0.5 \mathrm{~cm}$. 
Table 1

Parameters for yield curves in fig. 3

\begin{tabular}{|c|c|c|}
\hline & $\mathrm{ZnCdS}$ & $\mathrm{CdS}$ \\
\hline$N$, No. density of particles with $40 \AA$ diameter & $3.24 \times 10^{15} \mathrm{~cm}^{-3}$ & \\
\hline$N$, No. density of particles with $25 \AA$ diameter & & $6.39 \times 10^{15} \mathrm{~cm}^{-3}$ \\
\hline$d$, optical path length & $0.2 \mathrm{~cm}$ & $0.2 \mathrm{~cm}$ \\
\hline $\mathrm{OD}_{0}$, small-signal optical density at $308 \mathrm{~nm}$ & 0.715 & 0.330 \\
\hline$\sigma$, absorption cross-section at $308 \mathrm{~nm}$ & $25.4 \times 10^{-16} \mathrm{~cm}^{2}$ & $5.94 \times 10^{-16} \mathrm{~cm}^{2}$ \\
\hline$\alpha,=\sigma^{*} / \sigma[23]$ & 0.6 & 1 \\
\hline$\beta$, cf. eq. (4) of ref. [23] & 0.7 & 1 \\
\hline $\begin{array}{l}\sigma_{\text {el }} \text {, absorption cross-section of solvated } \\
\text { electrons at } 616 \mathrm{~nm}[26]\end{array}$ & $0.539 \times 10^{-16} \mathrm{~cm}^{2}$ & \\
\hline $\begin{array}{l}\sigma_{\mathrm{el}}, \text { absorption cross-section of solvated } \\
\text { electrons at } 720 \mathrm{~nm}[26]\end{array}$ & & $0.726 \times 10^{-16} \mathrm{~cm}^{2}$ \\
\hline$\sigma_{\mathrm{h}}$, absorption cross-section of holes at $616 \mathrm{~nm}$ & $0.013 \times 10^{-16} \mathrm{~cm}^{2}$ & \\
\hline$\sigma_{\mathrm{h}}$, absorption cross-section of holes at $720 \mathrm{~nm}$ & & $0.018 \times 10^{-16} \mathrm{~cm}^{2}$ \\
\hline$k_{\mathrm{T}}$, exciton trapping-rate constant & $35 \times 10^{12} \mathrm{~s}^{-1}$ & $300 \times 10^{12} \mathbf{s}^{-1}$ \\
\hline $\begin{array}{l}k_{\mathrm{A} 0}, \text { initial Auger emission-rate constant } \\
n_{\mathrm{el}}^{\max }, \text { maximum number of emitted electrons }\end{array}$ & $10^{15} \mathrm{~s}^{-1}$ & $10^{15} \mathrm{~s}^{-1}$ \\
\hline per particle & 16 & 6 \\
\hline
\end{tabular}

a) The high value for the Auger emission-rate constant $k_{\mathrm{A}}$ is relatively uncritical and merely indicates an initial quantum yield near one for the process.

tum-size calculations with a finite potential barrier as described in ref. [25]. From these curves we estimate the mean particle diameter $2 R$ for the CdS colloid to be $\approx 25 \AA$ and for the $\mathrm{ZnCdS}$ colloid to be $\approx 40 \AA$, which is in good agreement with our data from transmission electron microscopy. These values have to be compared with the excitonic diameter $2 a_{\mathrm{B}}$ of the bulk material ( $53 \AA$ for $\mathrm{CdS}$ and $40 \AA$ for $\mathrm{ZnCdS}$ ). The concentration of colloidal particles as well as the inferred absorption cross sections are collected in table 1.

Photophysical and photochemical changes after short UV pulse excitation were monitored by broadband transient absorption measurements in the wavelength range $370-750 \mathrm{~nm}$. The rise of all transient absorption bands occurred simultaneously within the time resolution of this measurement. Fig. 2 a shows transient spectra at 0.33 ps after excitation for CdS colloids in water and in isopropanol. Evidently, a strong additional absorption can be observed in the red spectral range in the case of the aqueous colloid. This is in agreement with findings in nanusecond flash phololysis experiments [27].

Fig. $2 b$ shows the difference between the two spectra of fig. $2 \mathrm{a}$ in comparison with the spectrum of a solvated electron reported in ref. [26]. The similarity between the two spectra as well as the analogy to nanosecond experiments allows us to conclude that in aqueous colloidal solution after intense UV pulse excitation we observe an "instantaneous" (within our temporal resolution of $250 \mathrm{fs}$ ) ejection of electrons. The almost structureless transient absorption in the range $440-720 \mathrm{~nm}$ in the case of the colloidal solution in isopropanol (or the difference between the transient spectrum of CdS colloid in water and the hydrated electron spectrum, respectively) can be assigned to photochemical changes due to trapped charge carriers. Again, this photochemical reaction (carrier trapping) is faster than $250 \mathrm{fs}$. The chemical nature of these photoproducts $\left(\mathrm{S}^{-}, \mathrm{e}^{-}\right.$associated with $\mathrm{Cd}^{++}$) has been discussed in $[23,28,29]^{\# 1}$. Here this is of no further interest, since on the time scale of interest up to 10 ps after excitation no significant absorption decay was observed in the red spectral range. Similar spectra have been observed for $\mathrm{Zn}_{x} \mathrm{Cd}_{1-x} \mathrm{~S}$.

\#1 It should be noted that a similar almost structureless transient absorption spectrum on the long wavelength side can also be observed with CdS and CdSSe microcrystallites embedded in a glass host [4]. 

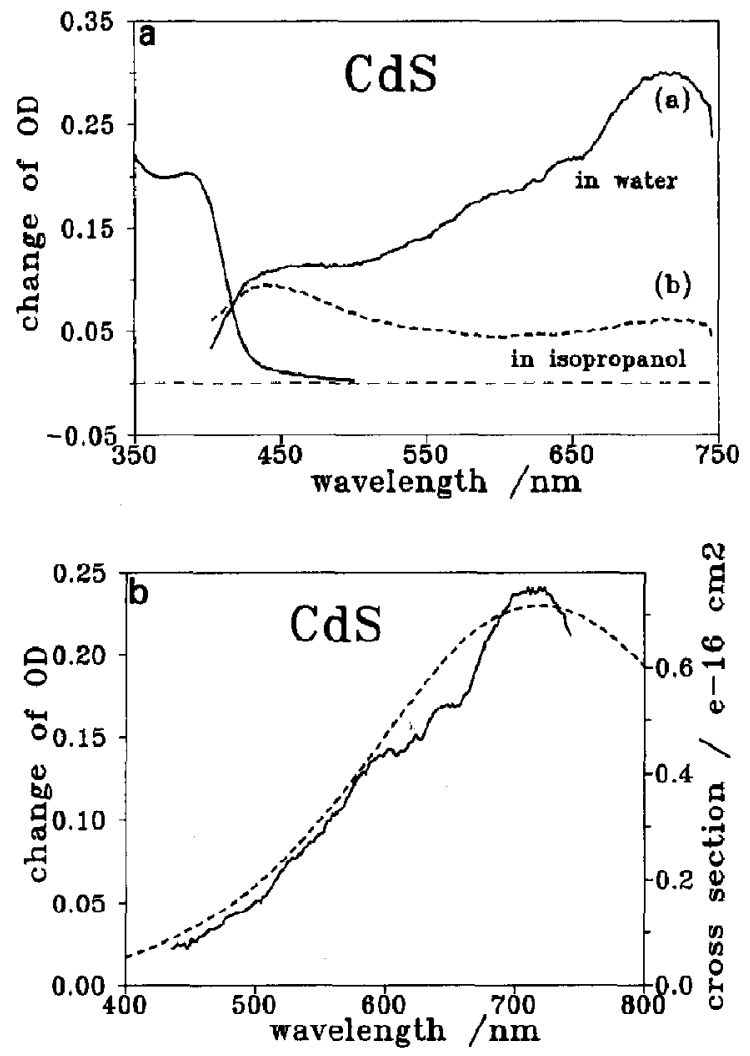

Fig. 2. (a) Transient absorption spectrum of $\mathrm{CdS}$ in isopropanol (a) and water (b) at $0.33 \mathrm{ps}$ after excitation by a $250 \mathrm{fs} \mathrm{UV}$ pulse. For comparison, the ground-state absorption spectrum of CdS is also shown for a sample path length of $0.2 \mathrm{~cm}$ as used in experiments. (b) Difference spectrum of colloidal CdS in water and and colloidal $\mathrm{CdS}$ in isopropanol at 0.33 ps after excitation by a 250 fs UV pulse. For comparison, the absorption spectrum of a solvated electron in water is shown [26] (dashed curve).

Fig. 3 shows the observed number of electrons per incident photon as a function of pump fluence as calculated from the maximum changes of optical density at $720 \mathrm{~nm}(\mathrm{CdS})$ and at $616 \mathrm{~nm}(\mathrm{ZnCdS})$ for $2 \mathrm{~mm}$ of the colloidal solution using the absorption cross-section of solvated electrons from ref. [26] (table 1) and the experimentally determined crosssections for the hole absorption (measurement in alcoholic colloids, table 1). Evidently, in the case of the CdS colloid, saturation of the ejection occurs at considerably higher pump fluences and only a third of the clectrons per colloidal particle is cjected. The solid lines in the figure represent the results of the model calculations (see below).

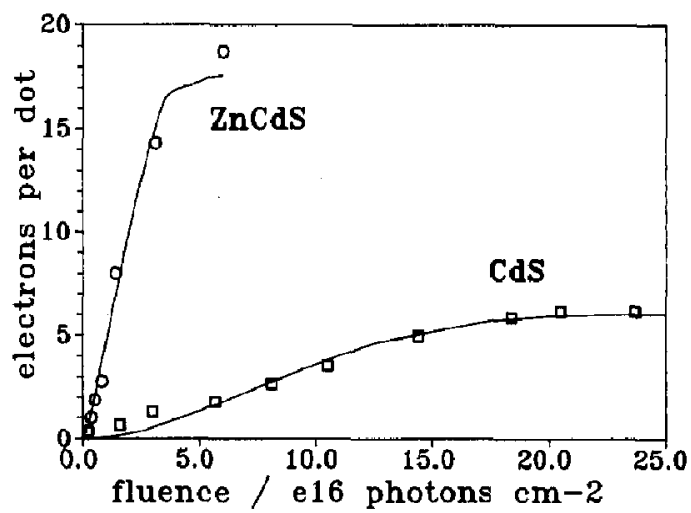

Fig. 3. Number of ejected electrons from aqueous CdS (hollow squares, obtained from change of optical density at $720 \mathrm{~nm}$ ) and $\mathrm{Zn}_{0.95} \mathrm{Cd}_{0.05} \mathrm{~S}$ colloids (hollow circles, obtained from change of optical density at $616 \mathrm{~nm}$ ) at $0.33 \mathrm{ps}$ after excitation by a $250 \mathrm{fs}$ UV pulse versus pump fluence at $308 \mathrm{~nm}$. Solid lines are the result of model calculations (see text).

\section{Discussion}

Since photochemical assignment of the broad-band absorption to trapped holes and trapped electrons has been discussed in ref. [23], we will focus here on the ejection of electrons in aqueous colloidal solution. Possible mechanisms of photoelectron generation in colloidal semiconductors have been discussed thoroughly in ref. [27]. Here, we will briefly review the main features with respect to our femtosecond experiments.

It was pointed out earlier $[27,30]$ that electrons cannot be photoemitted from CdS into water via a process in which only one photon is involved. This becomes evident by an inspection of the electrochemical potentials of the bulk semiconductor materials (CdS, $\mathrm{ZnS}$ ) [31] and the energy level of solvated electron [27] which are shown in fig. 4. The energy of the first excited ("excitonic") state is not high enough for an electron ejection.

A two-photon ionization as a possible process for the observed electron ejection is generally observed only when there is no one-photon absorption present. It should be also reflected in a characteristic dependence on the pump fluence, and in the case of negligible scrcening (CdS) no saturation should be observed. Measurements with nanosecond light pulses by some authors [27], however, showed an 


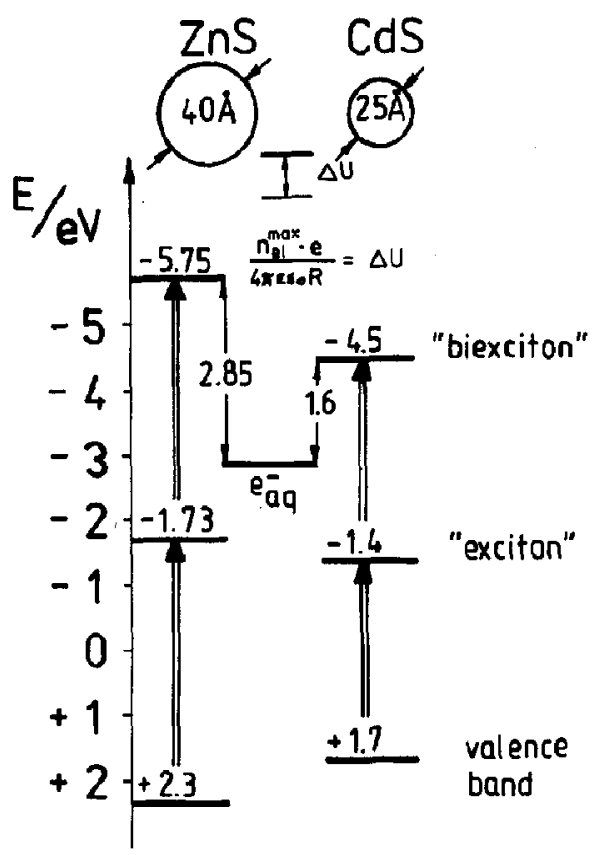

Fig. 4. Electrochemical potentials of bulk $\mathrm{CdS}$ and $\mathrm{ZnS}$ in water and potential energy of solvated electron [31]. Also shown are the "excitonic" and "biexcitonic" energy levels of the semiconductor colloids and schematically the reduction of the potential energy after electron ejection due to remaining positively charged excess holes.

almost linear dependence of the electron yield on the pump intensity up to $30 \mathrm{MW} / \mathrm{cm}^{2}$ and no saturation behavior: An Auger-like ejection process for electrons was thus proposed. Auger electron-emission processes have been observed also in (larger) semiconductor particles embedded in a glass host in nanosecond experiments [9]. In the latter experiments the Auger process was shown to greatly influence the relaxation process after light excitation as an additional recombination channel of the charge carriers, and therefore strongly decreases their lifetime.

Compared to these experiments, a much higher density of charge carriers occurs in our femtosecond investigations. If we bear in mind that in such tiny quantum-dots one electron-hole pair is already completely delocalized over the entire particle, it is evident that the spatial overlap with a second generated electron-hole pair is extremely large. This leads to an enhanced Auger emission rate. Such an electron-emitting state should be an analogue to a dou- bly-excited ("biexcitonic") state in a macrocrystal.

It might be argued that the electron emission could also occur from trapped states because trapping proceeds also within times shorter than 250 fs. However, due to the localization the spatial overlap of the charge-carrier wavefunctions is significantly lowered and consequently the probability for the Auger-like emission process will be greatly reduced. This is supported by the fact that in nanosecond transient absorption measurements (excitation pulse width 30 ns) [27] with pump fluences in the order of 150 photons per $\AA^{2}$ (compared to 10 photons per $\AA^{2}$ in our 250 fs experiments) the maximum electron yield for the hydrated electrons amounted to only 2-3\% \#2 compared to about $30 \%$ in our femtosecond experiments. The flux in the femtosecond experiments, however, is such that on the average every $10 \mathrm{fs}$ a photon is being absorbed by the quantum-dot, giving a certain probability for creating two or even more excitons before they are trapped or before they recombine.

In ref. [23] we have proposed a model which considers electron ejection, trapping and absorption of multiple photons (due to the high fluxes) (fig. 4), which we will briefly sketch here:

Absorption of a photon with absorption cross section $\sigma_{0}$ leads to the first excited ("excitonic") state a. This is followed by "excitonic" recombination or by trapping with a rate constant $k_{\mathrm{T}}$. In the case of strong pumping the "excitonic" state may absorb another photon (before trapping and recombination occur) leading to a doubly excited state $b$. Besides trapping and recombination another decay channel of this doubly excited state is given by the emission of an electron, with rate constant $k_{\mathrm{A}}$, leading to a deexcited particle with an excess hole. Since recombination of carriers proceeds on a picosecond timescale [23] the effect of an intense femtosecond pump pulse is to drive population up along the main diagonal in the scheme of fig. 5 creating particles having absorbed an average number $n_{\text {abs }}$ of photons and emitted an average number of electrons $n_{\mathrm{el}}$. This suggests a simpler energy-level scheme shown on the rhs of fig. 5 .

Rate equation calculation as in ref. [23] for this

\#2 From nanosecond control experiments as in ref. [27], with identical samples. 

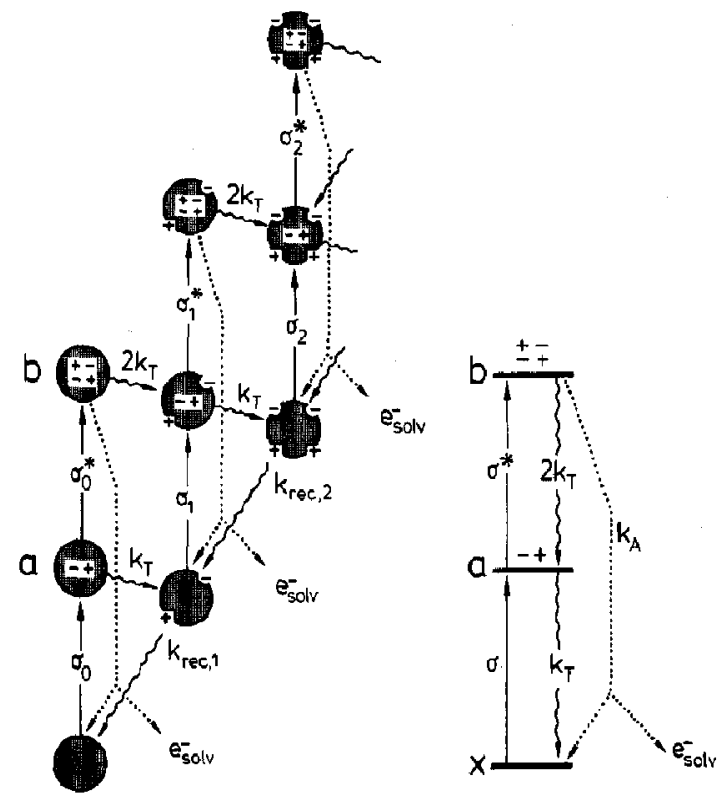

Fig. 5. Full and simplified formal rate model for multiphoton absorption by semiconductor microcrystallites.

model have been carried out to simulate the experimentally obtained electron yield curves (the number of electrons has been derived from experiment from the change of optical density in the red spectral range) (fig. 3). The parameters for best overall agreement with experiment are collected in table 1.

The decisive parameters of the model which have been varied in the course of calculation are:

- the trapping rate constant $k_{\mathrm{T}}$,

- and the maximum number of electrons which can be emitted by one particle $n_{\mathrm{el}}^{\max }$.

In particular, for CdS we find $k_{\mathrm{T}}=300 \mathrm{ps}^{-1}$, and $n_{\mathrm{el}}^{\max }=6$, whereas for the larger $\mathrm{ZnCdS}, k_{\mathrm{T}}=35 \mathrm{ps}^{-1}$, and $n_{\mathrm{el}}^{\max }=16$ have been obtained. $k_{\mathrm{T}}$ corresponds to an "exciton" lifetime of 3 fs for the CdS particle and 28 fis for the $\mathrm{ZnCdS}$ colloid, respectively. Calculating the transit time of an electron through such a small semiconductor particle (assuming an orbital velocity in an exciton of the corresponding bulk material), one finds characteristic times in the order of 10 fs [23]. We believe that the surface of the quantumdot is highly reactive and that it requires only a few orbits before the carriers are being trapped. The trapping possibly depends on the particular chemical preparation of the surface. Thus, such very fast trap- ping times, as well as the difference between CdS and $\mathrm{ZnCdS}$ (the CdS particle is significantly smaller) are indeed reasonable. However, the calculated values can only be considered as estimates. In particular, the value of $k_{\mathrm{T}}=300 \mathrm{ps}^{-1}$ for the CdS particle is already far beyond the limit where the rate equation description is applicable. We can take it therefore as a mere indication of almost instantaneous photochemical reaction on the surface.

Let us discuss now the second parameter of our model, the maximum number of electrons which can be ejected from such a particle, which differs by a factor of three for the two colloids. With every electron ejected from the doubly excited state, the remaining excess hole reduces the electrochemical potential [31] by $e / 4 \pi \epsilon_{0} \epsilon R$, where $\epsilon$ is the highfrequency dielectric constant (fig. 4). In this figure the energetic levels of the excitonic and biexcitonic states were calculated from the valence-band positions of the bulk materials and the spectral positions of the excitonic transitions of the investigated particles. The reduced potential decreases the driving force for the formation of hydrated electrons, which we incorporate into our model by an Auger rate constant $k_{\mathrm{A}}$ which depends on the number of electrons which have been ejected already, $n_{\mathrm{el}}$, and the total number of ejectable electrons, $n_{\mathrm{el}}^{\max }$ :

$k_{\mathrm{A}}=k_{\mathrm{A} 0}\left(1-n_{\mathrm{el}} / n_{\mathrm{el}}^{\max }\right)$ for $n_{\mathrm{el}}<n_{\mathrm{el}}^{\max }$

and

$k_{\mathrm{A}}=0$ otherwise.

Due to the larger particle diameter $2 R$ of the ZnCdS particle, the potential energy decrease with every ejected electron is smaller $(0.142 \mathrm{eV})$ compared to the $\mathrm{CdS}$ particle $(0.214 \mathrm{eV})$. The energy difference between the initial potential of the doubly excited ("biexcitonic") state and the solvated electron is larger ( $2.85 \mathrm{eV}$ ) for the $\mathrm{ZnCdS}$ colloid compared to $1.6 \mathrm{eV}$ for CdS particles. Thus, more electrons can be emitted by the ZnCdS particle in comparison to the smaller CdS microcrystallite. For the $\mathrm{ZnCdS}$ particle we can estimate the maximum number of electrons to be:

$n_{\mathrm{el}}^{\max }=2.85 \mathrm{eV} / 0.142 \mathrm{eV} \approx 20$

and for the CdS particle

$n_{\mathrm{el}}^{\max }=1.61 \mathrm{eV} / 0.214 \mathrm{eV} \approx 7.5$. 
From the fits with the parameters of table 1 , we find $n_{\mathrm{el}}^{\max }=16$ for $\mathrm{ZnCdS}$ and $n_{\mathrm{el}}^{\max }=6$ for $\mathrm{CdS}$, respectively. Thus, our experimental findings and the parameters of our model calculations are in good agreement with the values for the maximum number of electrons which can be ejected per dot derived from simple thermodynamic considerations.

\section{Conclusions}

We have monitored by subpicosecond spectroscopy the yield of photochemical products and the yield of solvated electrons from subpicosecond excitation of quantum-size $\mathrm{CdS}$ - and $\mathbf{Z n}_{0.95} \mathrm{Cd}_{0.05} \mathrm{~S}$-microcrystallites in alcoholic and aqueous colloidal solution, respectively. Both photochemical product formation (trapping) and electron ejection occurred within a time below our temporal resolution. The electron yield as a function of pump fluence was modelled in a simple kinetic scheme which involves the "exciton" lifetime prior to trapping, and a maximum number of electrons which can be emitted per particle. From the model calculations, "exciton" lifetimes (trapping times) in the order of 10-30 fs were obtained. The calculated maximum number of ejectable electrons from such a particle is in good qualitative agreement with thermodynamics. Our results suggest that the primary photophysical processes in such small quantum-dots differ considerably from the primary events in larger crystallites (in which $R$ is several times the excitonic radius) $[9,16]$. Further experimental and theoretical work, however, is needed to clarify the primary photophysical and photochemical events in such semiconductor quantum-dots. In particular, measurements are required in quantum-dots of different size, as well as with specific surface preparation, in order to elucidate the influence of the surface on the trapping and electron ejection. Also, a higher temporal resolution is needed to gain further evidence for the suggested model.

\section{Acknowledgement}

We are grateful to Professor F.P. Schäfer for his interest in this work, to the Deutsche Forschungs- gemeinschaft for support through the Leibnitz Prize Program. MK thanks the Max-Planck-Gesellschaft for a sholarship grant. Valuable discussions with Professor A. Henglein are gratefully acknowledged.

\section{References}

[ I] I. Broser and J. Gutowski, Appl. Phys. B 46 (1988) I.

[2] F. Henneberger, J. Puls, H. Rossmann, Ch. Spiegelberg, M. Kretzschmar and I. Haddad, Physica Scripta T13 (1986) 195.

[3] Ch. Spiegelherg, M. Kretzschmar, I. Puls and F. Henneberger, Phys. Stat. Sol. 150 b (1988) 769.

[4] M. Kaschke, B. Wilhelmi, V.D. Egorov, H.X. Nguyen and R. Zimmermann, Appl. Phys. B 45 (1988) 71.

[5] V.D. Egorov, P. Floegel, H,X. Nguyen and M. Kaschke, Phys. Stat. Sol. 146 b (1988) 351.

[6] J. Warnock and D.D. Awschalom, Appl. Phys. Letters 48 (1986) 426.

[7] M.C. Nuss, W. Zinth and W. Kaiser, Appl. Phys. Letters 49 (1986) 1717.

[8] G.R. Olbright, N. Peyghambrian, S.W. Koch and L. Banyai, Opt. Letters 12 (1987) 413.

[9] P. Roussignol, D. Ricard and C. Flytzanis, Appl. Phys. A 44 (1987) 285;

F. Rougemont, R. Frey, P. Roussignol, D. Ricard and C. Flytzanis, Appl. Phys. Letters 50 (1987) 1619.

[10] D.W. Hall and N.F. Borrelii, J. Opt. Soc. Am. B 5 (1988) 1650 .

[11] E.F. Hilinski, P.A. Lucas and Y. Wang, J. Chem. Phys. 87 (1988) 3435.

[12] F. Henneberger, U. Woggon J. Puls and Ch. Spiegelberg. Appl. Phys. B 46 ( 1988$) 19$.

[ 13 ] G.R. Olbright and N. Peyghambarian. Appl. Phys. Letters 48 (1986) 1184

[14] U. Woggon, F. Henneberger and M. Müller, Phys. Stat. Sol. 150 b (1988) 641.

[15] N. Peyghambarian and S.W. Koch, Rev. Phys. Appl. 22 (1987) 1711.

[16] V.S. Williams, G.R. Olbright, B.D. Fluegel, S.W. Koch and N. Peyghambarian, J. Mod. Optics 35 (1988) 1979.

[17] M. Kull, J.L. Coutaz, G. Manneberg and V. Grivickas, Appl. Phys. Letters 54 (1989) 1830.

[18] P.V. Kamat, T.W. Ebbesen, N.D. Dimitrijevic and A.J. Nozik, Chem. Phys. Letters 157 (1989) 384.

[19] L.E. Brus. IEEE J. Quantum Electron. QE-22 (1986) 1909.

[20] A. Fojtik. H. Weller, U. Koch and A. Henglein, Ber. Bunsenges. Physik. Chem. 88 (1984) 969:

A. Henglein, A. Fojtik and H. Weller, Ber. Bunsenges. Physik. Chem. 91 (1987) 441.

[21] A. Henglein. A. Kunar, E. Janata and H. Weller. Chem. Phys. Letters 132 (1986) 133;

Ch.-H. Fischer, H. Weller, A. Fojtik, C. Lume-Pereira, E. Janata and A. Henglein, Ber. Bunsenges. Physik. Chem. 90 (1986) 46; 
H. Weller, U. Koch, M. Gutiérrez and A. Henglein, Ber. Bunsenges. Physik. Chem. 88 (1984) 649.

[22] R. Rosetti, J.L. Elison, J.M. Gibson and L.E. Brus, J. Chem. Phys. 80 (1984) 4464;

R. Rosetti, R. Hull, J.M. Gibson and L.E. Brus, J. Chem. Phys. 82 (1985) 552; 83 (1985) 1406;

N. Chestnoy, R. Hull and L.E. Brus, J, Chem. Phys. 85 (1986) 2237.

[23] N.P. Ernsting, M. Kaschke, H. Weller, L. Katsikas, J. Opt. Soc. Am. B (1990), in press.

[24] N.P. Emsting and M. Kaschke, Appl. Phys. B, submitted for publication.

[25] H. Weller, H.M. Schmidt, U. Koch, A. Fojtik, S. Baral, A. Henglein, W. Kunath, W. Weiss and E. Diemann, Chem. Phys. Letters 124 (1986) 557.
[26] F.Y. Jou and G.R. Freeman, J. Phys. Chem. 83 (1979) 2383.

[27] M. Haase, H. Weller and A. Henglein, J. Phys. Chem, 92 (1988) 4706.

[28] S. Barral, A. Fojtik, H. Weller and A. Henglein, J. Am. Chem. Soc. 108 (1986) 375.

[29] A. Henglein, A. Kumar, E. Janata and H. Weller, Chem. Phys. Letters 132 (1986) 133.

[30] Z. Alfassi, D. Bahnemann and A. Henglein, J, Phys. Chem. 86 ( 1982$) 4656$.

[31]H. Gerischer, in: Photochemistry, photocatalysis, photoreactors, ed. M. Schiavello (Reidel, Dordrecht, 1985) pp. 39-116. 